\title{
Gut microbiome signatures of nursing home residents carrying Enterobacteria producing extended-spectrum $\beta$ - lactamases
}

Quentin Le Bastard ${ }^{1,2^{*}}$ (D), Guillaume Chapelet ${ }^{1,3}$, Gabriel Birgand ${ }^{1,4}$, Benjamin M. Hillmann ${ }^{5}$, François Javaudin ${ }^{1,2}$, Niki Hayatgheib' ${ }^{1}$, Céline Bourigault ${ }^{1,6}$, Pascale Bemer ${ }^{1,6}$, Laure De Decker ${ }^{3}$, Eric Batard ${ }^{1,2}$, Didier Lepelletier ${ }^{1,6}$ and Emmanuel Montassier ${ }^{1,2^{*}}$

\begin{abstract}
Background: The prevalence of extended beta-lactamase producing Enterobacteriaceae (ESBL-E) has been constantly increasing over the last few decades. These microorganisms that have acquired broad antibiotic resistance are now common human pathogens. Changes in the gut microbiome, induced by antibiotics or other drugs, enable expansion of these microorganisms, but the mechanisms are not yet fully understood.

Objectives: The main objective was to identify specific bacteria and functional pathways and genes characterizing the gut microbiome of nursing home residents carrying ESBL-E, using metagenomics.

Subjects and methods: We included 144 residents living in two different nursing homes. All fecal samples were screened for ESBL-E and gut microbiome was characterized using shallow shotgun metagenomic DNA sequencing.

Results: Ten nursing home residents were colonized by ESBL-E, namely Escherichia coli, Klebsiella pneumoniae and Enterobacter cloacae species, and were compared to non-carriers. We found that ESBL-E carriers had an alteration in within-sample diversity. Using a bootstrap algorithm, we found that the gut microbiome of ESBL-E carriers was depleted in butyrate-producing species, enriched in succinate-producing species and enriched in pathways involved in intracellular pH homeostasis compared to non-carriers individuals. Several energy metabolism pathways were overrepresented in ESBL-E carriers suggesting a greater ability to metabolize multiple microbiota and mucus layer-derived nutrients.

Conclusions: The gut microbiome of ESBL-E carriers in nursing homes harbors specific taxonomic and functional characteristics, conferring an environment that enables Enterobacteriaceae expansion. Here we describe new functional features associated with ESBL-E carriage that could help us to elucidate the complex interactions leading to colonization persistence in the human gut microbiota.
\end{abstract}

Keywords: Microbiome, Antibiotic resistance, Enterobacteriaceae, Metabolome

\footnotetext{
* Correspondence: quentin.lebastard@chu-nantes.fr;

emmanuel.montassier@chu-nantes.fr

'MiHAR lab, Université de Nantes, 44000 Nantes, France

Full list of author information is available at the end of the article
}

(C) The Author(s). 2020 Open Access This article is licensed under a Creative Commons Attribution 4.0 International License, which permits use, sharing, adaptation, distribution and reproduction in any medium or format, as long as you give appropriate credit to the original author(s) and the source, provide a link to the Creative Commons licence, and indicate if changes were made. The images or other third party material in this article are included in the article's Creative Commons licence, unless indicated otherwise in a credit line to the material. If material is not included in the article's Creative Commons licence and your intended use is not permitted by statutory regulation or exceeds the permitted use, you will need to obtain permission directly from the copyright holder. To view a copy of this licence, visit http://creativecommons.org/licenses/by/4.0/ The Creative Commons Public Domain Dedication waiver (http://creativecommons.org/publicdomain/zero/1.0/) applies to the data made available in this article, unless otherwise stated in a credit line to the data. 


\section{Introduction}

The spread of antimicrobial-resistant microorganisms is the main consequence of antibiotic overuse and misuse in human medicine and agricultural settings, leading to increased costs, treatment failures and mortality [1]. The rising prevalence of extended spectrum beta-lactamase producing Enterobacteriaceae (ESBL-E) worldwide is of particular concern [2]. Over the last decade in Europe, a significant increase in the prevalence of third-generation cephalosporin resistant Escherichia coli and Klebsiella pneumoniae was observed, from $0.1 \%$ in 1999 to $15 \%$ in 2017, and from $30.1 \%$ in 2011 to $37.2 \%$ in 2017 , respectively [3]. The prevalence of ESBL-E infections varies from region to region, with alarming rates in the Asia Pacific region [4]. As Enterobacteriaceae are among the most frequently isolated pathogens in bacterial infections in Europe, the spread of ESBL-E strains will lead to increased use of carbapenem, raising fears about the diffusion of carbapenems-resistant micro-organisms [5]. Numerous evidence supports the hypothesis that antibiotic resistance genes could spread between pathogenic and commensal bacteria through horizontal transfers and that the human gut microbiota stands as the main reservoir of ESBL-E [6, 7].

Changes in the gut microbiota composition, mostly in anaerobes, induced by antibiotic or nonantibiotic drugs such as proton pump inhibitors or oral antidiabetic medication, enable colonization by exogenous resistant bacteria [8, 9]. The enrichment in some species, such as Barnesiella have been reported to provide a colonization resistance against vancomycin resistant Enterococcus but the interactions within bacterial communities conferring resistance to colonization are not yet fully understood $[10,11]$. Two $16 \mathrm{~S}$ rRNA based studies identified specific taxonomic profiles associated with resistance to ESBL-E colonization in humans and a recent experimental work highlighted the role of short chain fatty acids (SCFA) mediated inhibition of ESBL-E [12-14]. To date, such observations have not been confirmed in humans. The main objective of this study was to identify specific bacteria and functional pathways or genes characterizing the gut microbiome of nursing home residents carrying ESBL-E, using a metagenomics approach. The identification of these features could help us to understand the mechanisms involved in the establishment and persistence of a microbiological environment favorable to colonization by ESBL-E.

\section{Subjects and methods}

\section{Subject recruitment and sample collection}

The following protocol was approved by the Nantes University institutional review board (IRB). This study was part of a point prevalence survey of ESBL-E and carbapenemaseproducing Enterobacteriaceae gastrointestinal carriage conducted from July to August 2016 [15]. The study population consisted of 144 elderly residents from two public nursing homes (312 residents) in Nantes (Western France). Residents were enrolled according to their (or their surrogate) ability to provide written consent. Age, morphological data, comorbidities, current and previous medications including antibiotics, were collected from medical records.

Fecal samples were collected by the nursing staff by swabbing freshly emitted stools (Copan or FecalSwab, Copan Diagnostics, Murrieta, CA, USA) in each participant for microbiological identification of ESBL-E (see Supplementary materials for laboratory methods). Samples were addressed and managed in the laboratory of Bacteriology, Nantes University Hospital, for identification of ESBL, according to CLSI guidelines. Samples were vortexed and cultured for $24 \mathrm{~h}$ on specific chromogenic agar culture medium (ESBL-chromID, Biomérieux, France). Colonies were identified using mass spectrometry (MALDI-TOF MS method, Bruker Daltonics, Germany). ESBL-E phenotypes were confirmed by detection on disc diffusion tests (MASTDISCSTM D72C ID ESBL test, Mast Diagnostics, Merseyside, UK). Samples for metagenomic analysis were immediately stored at $4{ }^{\circ} \mathrm{C}$ in RNA later (Fisher scientific, Waltham, MA, USA) during $24 \mathrm{~h}$ and then at $-80^{\circ} \mathrm{C}$ until sequencing.

\section{Shotgun metagenomics analysis}

DNA extraction, amplification, and sequencing were performed at the University of Minnesota Genomics Center (UMGC). DNA was extracted from frozen fecal samples using the MO BIO PowerSoil DNA Isolation Kit (MO BIO, Carlsbad, CA, USA) and quantified using the Quant-iT PicoGreen dsDNA Assay kit (Fisher scientific, Waltham, MA, USA). A shallow shotgun sequencing of total stool DNA was applied using Illumina HiSeq platform. Paired-end sequencing was performed using $2 \times 250$ base-pair reads following manufacturer recommendations. Libraries were prepared using Illumina barcodes (TruSeq DNA Sample Prep kit) and KAPA biosystems reagents (KAPA Library Preparation kit). The resulting DNA libraries were amplified by PCR using KAPA HiFi polymerase (Roche, Bâle, Switzerland) [16]. Reads were aligned up to species level against the comprehensive NCBI RefSeq database release 87 [17] using BURST aligner (v0.99.7) with a threshold of $97 \%$ identity $[18,19]$. All matched bacterial species were included. Functional annotations were obtained using the HUMAnN2 [20] pipeline (v0.11.2) against KEGG bacteria and archaea genome database (v56). Statistical analysis were performed in the $\mathrm{R}$ (version 3.4.1) environment.

Within-samples taxonomic and functional biodiversity (alpha diversity) were measured using the QIIME2 (version 2018.4) diversity script after multiple rarefactions at 
species level (210,696 sequences/samples) [21]. Relative abundance were computed using the QIIME2 featuretable script. CAZyme annotations were obtained using Diamond (version v0.9.24.125, default parameters $-\mathrm{k} 25$ and -e 0.001) and the CAZy database (release 07/26/ 2019) [22, 23]. We compared Chao1 and unique observed-species between groups using a nonparametric two-sample mean difference test with 999 Monte Carlo permutations. We quantified taxonomic and functional biodiversity differences between samples (beta-diversity) by computing Bray Curtis distances with the vegan $R$ package (version 2.5-1) between all pairs of samples and compared groups using ANOSIM with 999 Monte Carlo permutations. Then, we identified differences in taxonomic and functional items abundance between groups by estimating $\log 2$-fold change with the likelihood ratio test included in the DESeq2 package (v1.18.1) [24]. We used a model fitting a local regression of log dispersion over $\log$ base mean with a negative binomial distribution. $P$-values were adjusted for multiple testing using the False Discovery Rate (FDR) (Benjamini-Hochberg adjustment).

We used a bootstrap algorithm with 200 iterations to compare ESBL-E carriers and non-carriers. In detail, we randomly selected 200 times 40 ESBL-E non-carriers samples that were compared to the ESBL-E carriers $(n=$ 10) with DESeq2. All taxonomic and functional items bootstrapped mean log2-fold changes with adjusted $p$ values $<0.10$ were considered as statistically significant and plotted. We considered a robust signature, an item that was significantly different between 2 groups in at least $70 \%$ of all repeated procedures $[25,26]$.

The associations of individual species to collected covariates were assessed using MaAsLin (v0.0.4) with default settings in $\mathrm{R}$ environment [27]. In each analysis, the false discovery rate was controlled at FDR corrected $p$ value 0.1 using the $\mathrm{R}$-package $\mathrm{Q}$-value (v3.10). (See supplemental methods for more details).

\section{Results}

\section{Study population}

We enrolled 144 of the 312 residents of the two nursing home. Ten (6.9\%) of them were confirmed to carry ESBL-E. Microbiological analysis identified seven Escherichia coli, two Enterobacter cloacae and one Klebsiella pneumoniae. The relative abundance of these species was not significantly different between carriers and noncarriers (Mann-Whitney U test, ESBL-E carriers versus non-carriers for E. coli, E. cloacae and K. pneumoniae relative abundances, all $p$ values $>0.05$ ) (Supplementary Figure S1). There were no significant differences in baseline characteristics between carriers and non-carrier subjects, including antibiotic exposure (Table 1). Prevalence of ESBL-E carriage were not different between the two
Table 1 Baseline characteristics of ESBL-E carriers and noncarriers among residents of the two nursing homes. Univariate analysis were performed using Wilcoxon signed rank test

\begin{tabular}{|c|c|c|c|}
\hline \multirow[b]{2}{*}{ Baseline Characteristics } & \multicolumn{2}{|c|}{ No. of residents (\%) } & \multirow{2}{*}{$\begin{array}{l}P \\
\text { value }\end{array}$} \\
\hline & $\begin{array}{l}\text { ESBL-E carriers } \\
(n=10)\end{array}$ & $\begin{array}{l}\text { Non carriers } \\
(\boldsymbol{n}=134)\end{array}$ & \\
\hline Sex (females) & $7(70)$ & $90(67)$ & 0.8 \\
\hline Age, mean (SD) & $85.6( \pm 5.82)$ & $86.13( \pm 6.61)$ & 0.53 \\
\hline Dementia & $7(70)$ & $78(58)$ & 0.48 \\
\hline Parkinson disease & $0(0)$ & $9(7)$ & 0.4 \\
\hline Diabete & $0(0)$ & $30(22)$ & 0.095 \\
\hline Peptic ulcer disease & $1(10)$ & $13(10)$ & 0.98 \\
\hline Chronical kidney disease & $1(10)$ & $14(10)$ & 0.96 \\
\hline Long term stay (> 24 months) & $5(50)$ & $94(70)$ & 0.188 \\
\hline \multicolumn{4}{|c|}{ Medications during past 3 months } \\
\hline Oral antidiabetic medication & $1(10)$ & $10(7)$ & 0.78 \\
\hline Anti-acid medication & $4(40)$ & $26(19)$ & 0.124 \\
\hline Broad spectum pennicilin & $4(40)$ & $41(31)$ & 0.54 \\
\hline Anxiolytic medication & $7(70)$ & $98(73)$ & 0.82 \\
\hline Antidepressant medication & $3(30)$ & $75(56)$ & 0.11 \\
\hline Antiepileptic medication & $1(10$ & $24(18)$ & 0.53 \\
\hline Proton-pump inhibitors & $1(10)$ & $5(4)$ & 0.35 \\
\hline
\end{tabular}

nursing homes (Fisher's exact test, $p$ value $>0,05$ ). Among ESBL-E carriers, four were exposed to broad spectrum penicillin during 7 days. As all residents were housed in two nursing homes from the same University hospital, diet, hygiene and care was performed to a standardized practice by the same medical and nursing staff and meals were prepared in the same facility.

\section{Diversity of the gut microbiome is lower in ESBL-E carriers}

We obtained the mean 308,696 sequences per samples, providing enough depth to reach species-level [16].

Comparison of within-samples species diversity (alpha-diversity), using Chaol and unique observed species indexes, showed a less diverse gut microbiome in ESBL-E carriers (Mann-Whitney U test, ESBL-E carriers versus non-carriers, Chao1 and unique observed species, $p$ value $<0.001$ and $p$ value $<0.01$, respectively, Fig. 1$)$. Principal-coordinates analysis ( $\mathrm{PCoA})$ of Bray Curtis distances revealed that carriers and non-carriers also harbored a slight but significantly distinct gut microbial compositions. The $\mathrm{R}$ value, close to 0 suggests the existence of an important overlap area between the 2 groups (analysis of similarities, ANOSIM, $\mathrm{R}=0.177, p$ value $=$ 0.048 , Supplementary Figure S2). Carriers and noncarriers were not clustered according to their functional architecture (ANOSIM, $\mathrm{R}=0.1, p$ value $=0.15$ ), but we observed a trend in increased functional biodiversity in 


\section{Species biodiversity}
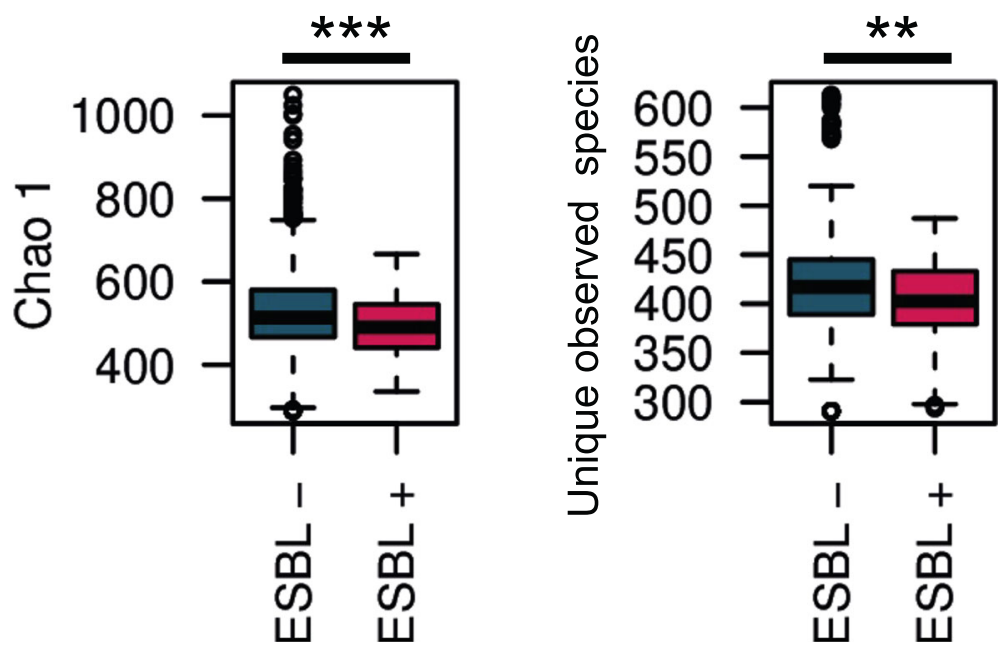

Fig. 1 Alpha-diversity indices in gut microbiomes of extended spectrum beta-lactamase producing Enterobacteriaceae (ESBL-E) carriers (ESBL+) and non-carriers (ESBL-), based on species. Whiskers in the boxplot represent the range of minimum and maximum alpha diversity values within a population, excluding outliers. Monte-Carlo permutation t-test: ${ }^{*} p$ value $<0.05$; ${ }^{* *} p$ value $<0.01$; and ${ }^{* * *} p$ value $<0.001$. Boxplots denote top quartile, median, and bottom quartile. (ESBL-E) carriers had significantly lower microbial richness compared with non-carriers, using Chao1 and unique observed species ( $p$ value $<0,001$ and $p$ value $<0,01$, respectively)

carriers $(2,129,865$ sequences/samples, Mann-Whitney U test, ESBL-E carriers versus non-carriers, Chao1 and unique observed species, $p$ value $<0.01$ and $p$ value $<$ 0.175, respectively, Supplementary Figure S3). Importantly, we did not find any significant association between other covariables and interindividual microbial distance, including sex, age, length of stay, comorbidities and medications.

\section{ESBL-E carriers gut microbiome is enriched in Bacteroides and Prevotella}

To identify a taxonomic signature associated with ESBL-E carriage, we compared bacterial communities of carriers and non-carriers at genus and species levels using DESeq2 with bootstrap iterations. The great majority of genera overrepresented in the gut microbiome of ESBL-E carriers mapped to Bacteroidales spp. and Clostridiales spp. orders (respectively 4 and 2 of 9). In particular, samples from carriers showed a more than 2 mean log2fold higher proportion of Megasphaera spp., Alloprevotella spp. (FDR corrected $p$ value $<0.10$, in $70 \%$ or more bootstrapping iterations). Less frequently, Prevotella spp. and Bacteroides spp. appeared increased in ESBL-E carriers (FDR corrected $p$ value $<0.10$, in $60 \%$ or more bootstrapping iterations). The representation of Pseudomonas spp. and Johnsonella spp. genera appeared strongly decreased with a mean log2fold change below -4 (FDR corrected $p$ value $<0.10$, in
70 and $60 \%$ or more bootstrapping iterations respectively) (Fig. 2a, Supplementary Table S1).

More precisely, bacterial species composing the community in ESBL-E carriers were significantly enriched in 8 species. Bifidobacterium animalis showed a strongly increased abundance of 6 mean log2fold change and three Prevotella species were increased over 2 mean log2fold change ( $P$. corporis, $P$. timonensis and $P$ sp. P4-76). Species belonging to Bacteroides spp. genera represented a large proportion of the overrepresented individual bacteria in these residents (B. faecichinchillae, B. coprophilus, B. ovatus). The lower proportion of Johnsonella spp. genera in ESBL-E carriers was mostly represented by a lower abundance of J. ignava and Pseudomonas spp. genera by Pseudomonas aeruginosa (FDR corrected $p$ value < 0.10 , in at least 70 and $60 \%$ or more bootstrapping iterations respectively). Three individual bacterial species showed a strongly decreased abundance below - 3 mean log2fold change, namely Clostridium hylemona and Collinsella tanakaei (FDR corrected $p$ value $<0.10$, in at least $70 \%$ or more bootstrapping iterations) and less frequently Bifidobacterium adolescentis (FDR corrected $p$ value $<0.10$, in at least $60 \%$ or more bootstrapping iterations) (Fig. 2b, Supplementary Table S2).

As many parameters may influence the composition of the gut microbiome, we used the MaAslin pipeline to test for association between baseline characteristics and 


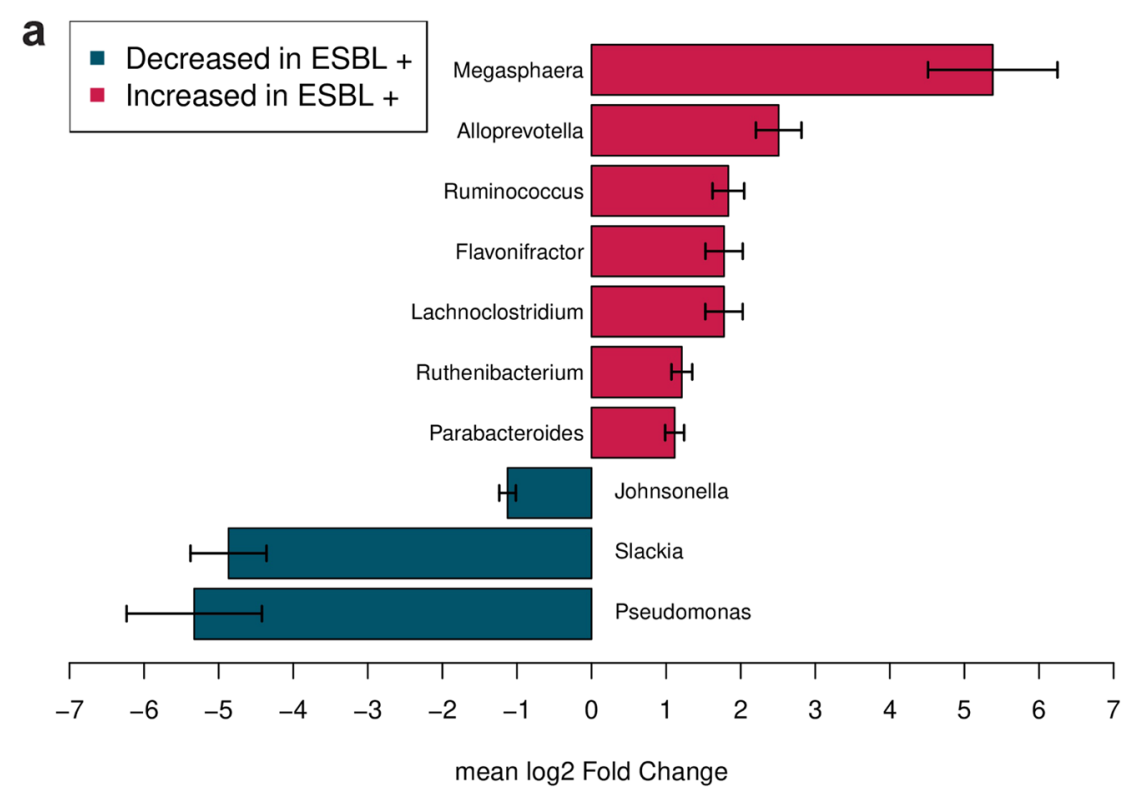

b



Fig. 2 a Microbes that differentiate Enterobacteriaceae (ESBL-E) carriers and non-carriers at genus level, using DESeq2 with bootstrap iterations. The bootstrap model consists in 200 random selections of non-carriers $(n=40)$. We represented the genera that are significantly different between ESBL-E carriers and non-carriers in more than $70 \%$ of the iterations, with a FDR corrected $p$ value $<0.10$. Genera with increased abundance in ESBL-E carriers are represented in red, and genera with decreased abundance are represented in blue, $\mathbf{b}$ Microbes that differentiate Enterobacteriaceae (ESBL-E) carriers (ESBL+) and non-carriers (ESBL-) at species level, using DESeq2 with bootstrap iterations. The bootstrap model consists in 200 random selections of non-carriers $(n=40)$. We represented the species that are significantly different between ESBL-E carriers and non-carriers in more than $70 \%$ of the iterations, with a FDR corrected $p$ value $<0.10$. Species with increased abundance in ESBL-E carriers are represented in red, and species with decreased abundance are represented in blue

taxonomical composition of the gut microbiome of all residents. We did not find any significant association between age, sex, length of stay, medications, comorbidities and taxonomical composition of the gut microbiome.
The gut microbiome of ESBL-E carriers is enriched in carbohydrate metabolism pathway genes

The analysis of functional modules harbored by the gut microbiome showed robust differences in relative 
abundance of 8 KEGG modules between ESBL-E carriers and non-carriers (FDR corrected $p$ value $<0.10$, in $70 \%$ or more bootstrapping iterations) (Fig. 3, Supplementary Table S3). All functional pathways identified were overrepresented in ESBL-E carriers and were involved in carbohydrate metabolism, amino-acid and branchedchain amino acid metabolism and environmental information processing. Among them, KEGG modules involved in cysteine biosynthesis (M00338), glutamate transport (M00233), sulfonate transport (M00436), Nacetylglucosamine biosynthesis (M00267 and M00019) and isoleucine biosynthesis (M00570) were the most frequently significant. We identified a total of 50 KEGG orthologies (KOs) with modified relative abundance between carriers and non-carriers, which were all overrepresented in ESBL-E carriers gut microbiome (FDR corrected $p$ value $<0.10$ in $70 \%$ or more bootstrapping iterations) (Supplementary Figure S4 and supplementary Table S4). Finally, to further investigate carbohydrate metabolism, we searched for a signature in CAZymes composition. Nine CAZymes had a modified relative abundance between carriers and non-carriers, all overrepresented in ESBL-E carriers gut microbiome (FDR corrected $p$ value $<0.10$ in $70 \%$ or more bootstrapping iterations) (Supplementary figure and supplementary Table S5).

\section{Discussion}

The colonization of the human gut microbiome by antibiotic-resistant Enterobacteriaceae strains is of peculiar concern in clinical practice, as it exposes to the risk of spread within the community, increasing the subsequent risk of disseminated infections, including bacteremia [28]. Here, we focused on describing the taxonomical and functional signature of ESBL-E carriers gut microbiome. When compared to non-carriers, the community is primarily characterized by a decreased abundance in SCFAs-producing bacteria, an increased ability to produce succinate, and an increased ability to metabolize carbohydrates derived from the mucus layer. Our understanding of the interactions contributing to colonization by resistant germs within the microbiota is limited. Our results confirm in humans the existence of mechanisms observed in vitro that could be future therapeutic targets for preventive or curative strategies. We did not identify any other parameters influencing the composition of the gut microbiome in this cohort. To our knowledge, this is the first study aimed to explore the specific patterns of the gut microbiome associated with ESBL-E carriage using shotgun metagenomic sequencing, providing a deeper description of bacterial communities and functional pathways than previous works in the field.

Overall, we found that our results are consistent with previous experimental observations and highlight several mechanisms that may play a key role in the expansion of antimicrobial-resistant in the human gut microbiome (Fig. 4). Among them, SCFAs production seems to be the most crucial function of the gut microbiota in terms of providing colonization resistance against ESBL-E [14]. In this cohort, we observed that the gut microbiome of ESBLE-E carriers was characterized by a lower

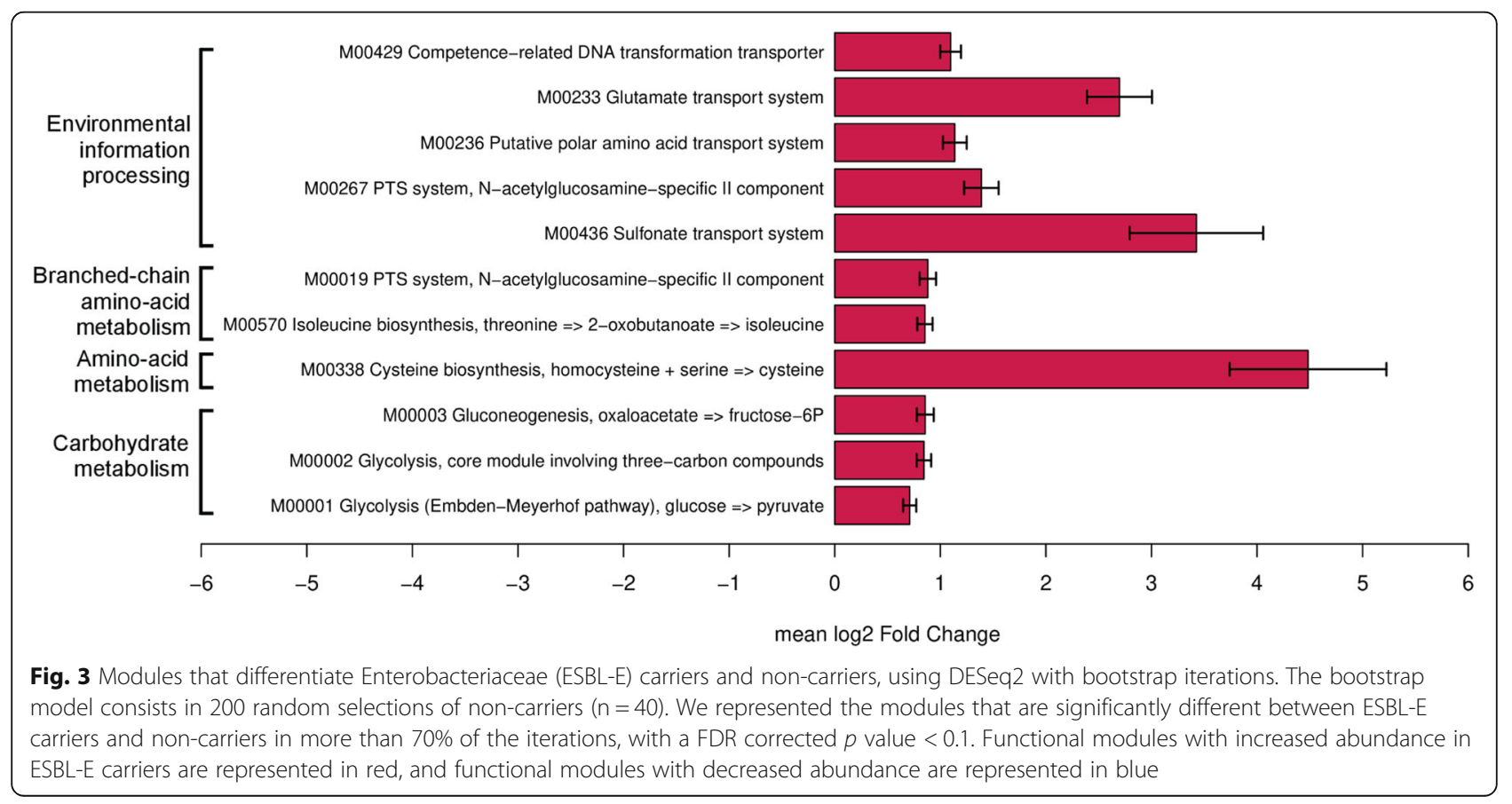




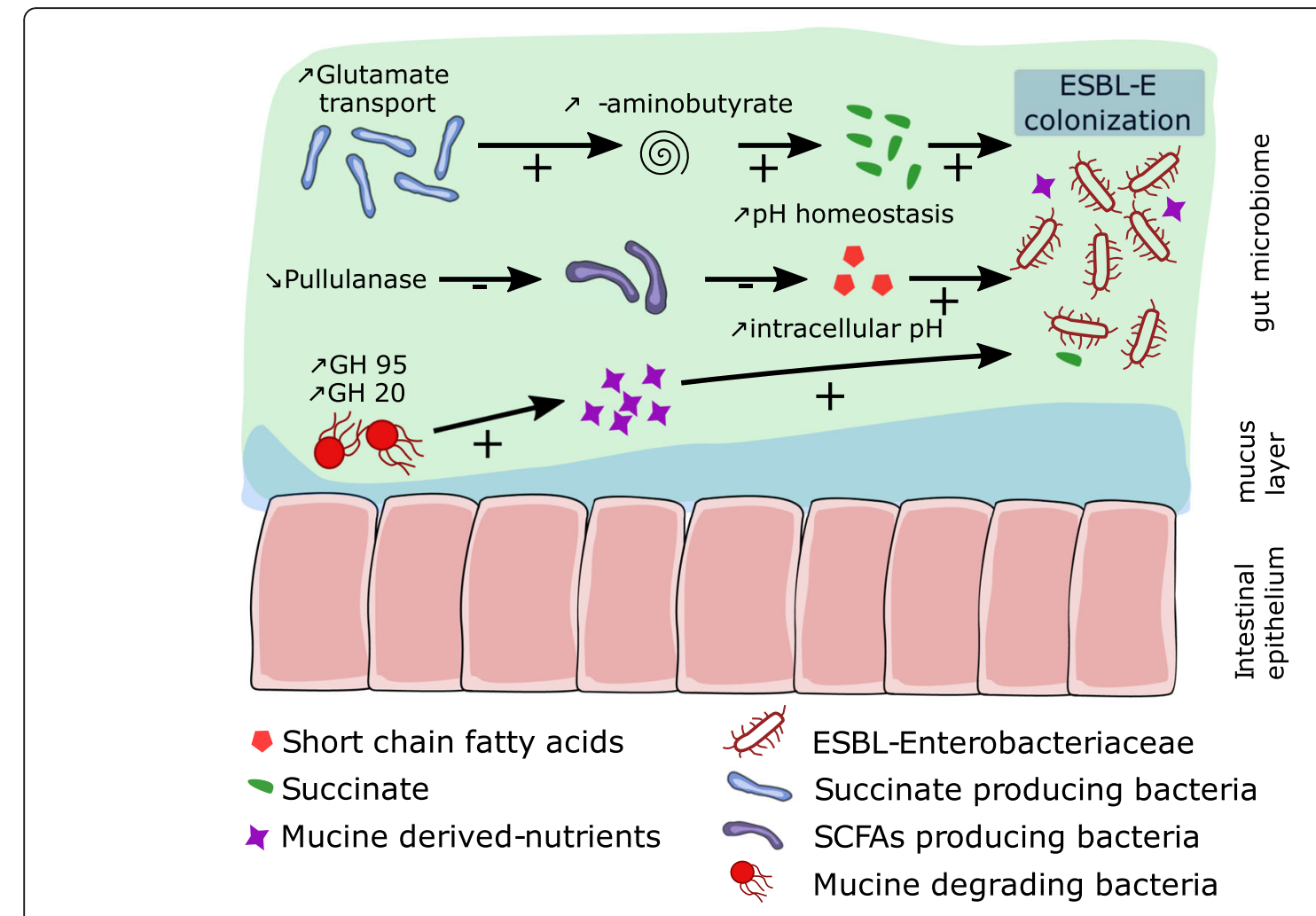

Fig. 4 Proposed mechanisms that may play a role in the expansion of antimicrobial-resistant Enterobacteriaceae in the human gut microbiome

abundance of SCFAs producing bacteria. A large proportion of the bacteria we found depleted in the gut microbiome of ESBL-E carriers, namely Clostridium hylemonae, Collinsella tanakaei, Johnsonella ignava, and Bifidobacterium adolescentis have been identified as being directly involved in SCFAs production such as acetate, propionate, and butyrate. This supports the hypothesis that a depletion in SCFAs producing species may be associated with ESBL-E colonization and persistence in the gut [2931]. Moreover, the only under-represented metabolic feature in ESBL-E carriers was pullulanase (K01200), a glucanase that degrades pullulan, which has been reported to stimulate butyrate production by promoting the growth of Bifidobacteria species [32]. A recent experimental work highlighted that the maintenance of an acidic environment coupled with production of high concentration of SCFAs by the colonic microbiota promote the clearance of EBLS-E [14]. The authors demonstrate that SCFAs can directly trigger intracellular acidification of ESBL-E to counter the competitive edge that $\mathrm{O}_{2}$ and $\mathrm{NO}_{3}$ respiration confer upon Enterobacteriaceae during expansion. Here, the most significantly overrepresented species in ESBL-E carriers was Bifidobacterium adolescentis. This bacterium was also found increased in the gut microbiome of travelers who eradicate ESBL-E colonization when compared to travelers who did not suggesting it could play a key role in colonization clearance of antibiotic resistant bacteria. In the same way, travelers who did not cleared ESBL-E colonization harbored a significantly higher proportion of Bacteroides sp. in their gut microbiome such as ESBL-E carriers in our study, suggesting a role for this genus in colonization persistence of antibiotic resistant bacteria [33].

In our analysis of the functional profile of ESBL-E carriers, we found evidences suggesting an increased capacity of succinate production. There were a greater abundance of gene that function in the transport of glutamate (M00233, K10008, K10007 and K10006) in ESBL-E carriers suggesting an increased glutamate metabolism. Glutamate is involved in the biosynthesis of several proteins and mostly metabolized via the GABA shunt pathway. More specifically, the production of $\gamma$ aminobutyrate through a decarboxylation reaction of glutamate consumes protons which are subsequently removed from the environment. The production of succinate from glutamate by Glutamate Decarboxylase has been reported to be implicated in bacterial acid tolerance by facilitating the homeostasis of intracellular $\mathrm{pH}$ [34]. These observations were consistent with the enrichment of the intestinal microbiome of ESBL-E carriers with bacteria of the genera Prevotella and Bacteroides. Both are known to produce succinate, suggesting that their overrepresentation in the microbiota could lead to greater availability of succinate [34, 35]. Some bacterial pathogens have been described to 
metabolize microbiota-derived succinate for their own benefit. In Salmonella typhimurium, succinate metabolism contributes to efficient colonization of the intestinal lumen, and in Clostridium difficile it promotes the onset of infection after antibiotic treatment [36, 37]. Overall, these data suggest that modulations of the gut microbiome leading to increased succinate production and a reduced SCFAs availability could provide a favorable environment for ESBL-E colonization.

Our data also show that the gut microbiome of ESBLE carriers expresses a greater diversity of energy metabolism pathways with an overrepresentation of gluconeogenesis (M00003), glycolysis (M00001, M00002) and pentose-phosphate pathway (K07404). The greater abundance of modules that function in amino-acid metabolism, especially isoleucine and methionine (M00570, M00019, M00338) and gluconeogenesis (M00003) suggests an intense energy metabolism activity involving alternative pathways that use amino-acids instead of carbohydrates to generate glucose. This type of mechanism has been observed in urinary tract infections due to Escherichia coli [38]. The increased diversity of genes operating in energy metabolic pathways may reflect a greater ability to metabolize multiple microbiota-derived nutrients. This could provide a growth facility for certain species such as Enterobacteriaceae and promote colonization as previously described with gluconeogenesis for the maintenance of Escherichia coli O157:H7 in the microbiota [39]. We observed an increased abundance of glycoside hydrolase genes in the microbiome of ESBL-E carriers. In particular, the overrepresentation of the glycoside hydrolase genes GH 95 and GH 20 suggests that the microbiome of ESBL-E carriers exhibits increased mucin degradation capacity. Mucin degradation results in increased availability of host-derived sugar, such as fucose, which has been shown to facilitate postantibiotic expansion of enteric pathogens [40, 41]. Thus, the gut signature of ESBL-E carriers is characterized by a greater ability to metabolize multiple microbiota and mucus layer derived carbohydrates. This suggests that colonization is enabled by the establishment of a favorable nutrient environment.

Finally, metabolic pathways suspected to be part of the bacterial mechanisms involved in colonization were also overrepresented in ESBL-E carriers. Among them, we identified an increased abundance of pathways involved in sulfur cycling (M00436, K15553, K15555), supposed to increase bacterial resistance to antibiotics and to protect them from reactive oxygen species [42].

\section{Limitations}

Our study has several limitations, the main one being the low prevalence of ESBL-E colonization in our cohort, the reason why we used bootstrap iterations. This has also limited the statistical power when comparing of baseline characteristics of colonized and non-colonized residents. Second, although subjects included in our cohort were exposed to controlled medication and received the same dietary, the same hygiene and care support, all the parameters that may affect gut microbiome composition, such as visits by outside individuals, were not controlled. Third, this study only provides information on the characteristics of the gut microbiome allowing for persistence of colonization but neither explains the initial changes that occurs nor the mechanisms leading to the acquisition of these resistant strains, as longitudinal design could have done. Also, because this work is based on DNA sequence reads analysis, it only provides informations about metabolic coding capacities but do not prejudge that these sequences are transcribed into actual functions. The use of shallow shotgun provides an information about species communities and functional capacities but is not as accurate as deep-shotgun. Thus, poorly represented species or functional genes may have been missed with this strategy. Further investigations, including transcriptomic, metabolomic analysis and multiple time points are needed to confirm the upregulation of these metabolic pathways and the increased availability of succinate in the gut lumen of ESBL-E carriers.

\section{Conclusions}

In this work we described the gut-microbiome signature of ESBL-E carriers. Our observations highlight several mechanisms involved in the colonization of the human gut microbiota by ESBL-E in nursing home residents. This includes a modification in acidity regulation with a decreased SCFAs production and a greater ability to regulate intracellular $\mathrm{pH}$ through an increased succinate availability providing an electron-donor for respiratory chain. The greater availability of microbiota-derived nutrients derived from the mucus layer degradation, combined with increased energy metabolism pathways, could provide a growth facility for ESBL-E and promote colonization. Overall, these mechanisms confer an environment enabling the establishment and survival of Enterobacteriaceae in the gut. These observations, especially the role of succinate, require further investigation, including metabolomic, and could help us to elucidate the complex interactions leading to colonization persistence in the human gut microbiota. Future works should focus on setting up a metabolomics pilot study, including multiple time point samplings, to investigate the role of succinate. Tailored shifts in the gut microbiota based on the administration of complex consortia of bacteria able to increase SCFAs production and to decrease succinate availability might prevent the colonization and contain the spread of antimicrobialresistant microorganisms in humans. 


\section{Supplementary information}

Supplementary information accompanies this paper at https://doi.org/10. 1186/s13756-020-00773-y.

Additional file 1. Supplementary methods and figures.

Additional file 2. Supplementary Table 1. Genus that differentiate carriers and non-carriers using DESeq2 with bootstrap iterations.

Additional file 3. Supplementary Table 2. Species that differentiate carriers and non-carriers using DESeq2 with bootstrap iterations.

Additional file 4. Supplementary Table 3. KEGG modules that differentiate carriers and non-carriers using DESeq2 with bootstrap iterations.

Additional file 5. Supplementary Table 4. KEGG KO that differentiate carriers and non-carriers using DESeq2 with bootstrap iterations.

Additional file 6. Supplementary Table 5. CAZyme that differentiate carriers and non-carriers using DESeq2 with bootstrap iterations.

\section{Abbreviations}

ESBL-E: Extended spectrum beta-lactamase producing Enterobacteriaceae; GH: Glycoside hydrolase; GT: Glycosyl transferase; KOs: KEGG orthologies; SCFA: Short chain fatty acids

\section{Acknowledgements}

The authors are grateful to the Genomics and Bioinformatics Core Facility of Nantes.

\section{Authors' contributions}

EM, EB, DLP, LDD: design of study; FJ, CB, GB, GC: acquisition of data; PB, CB, $\mathrm{NH}$ : bacteriological analysis; EM, QLB, HB: microbiome and statistical analysis. QLB, EM: draft of the manuscript. All authors read and approved the final manuscript.

\section{Funding}

This work was supported by the University of Nantes.

\section{Availability of data and materials}

The datasets generated during the current study are available in the NCBI Sequence Read Archive under BioProject ID PRJNA531316.

\section{Ethics approval}

The study protocol was approved by the IRB of the Nantes University Hospital and was conducted in compliance with the Helsinki Declaration and to local legislation.

\section{Consent for publication}

All patient information was anonymized at source and unique ID codes were used to identify cases, as requested by the IRB of the Nantes University Hospital.

\section{Competing interests}

The authors declare that they have no competing interests.

\section{Author details}

${ }^{1}$ MiHAR lab, Université de Nantes, 44000 Nantes, France. ${ }^{2}$ Department of Emergency Medicine, CHU Nantes, Nantes University Hospital, 44000 Nantes, France. ${ }^{3}$ Pole de gérontologie clinique, Nantes University Hospital, 44000 Nantes, France. ${ }^{4}$ Regional Infection Control Centre, Pays de la Loire, Nantes, France. ${ }^{5}$ Department of Computer Science and Engineering, University of Minnesota, Minneapolis, Minnesota, USA. ${ }^{6}$ Bacteriology and Infection Control Department, Nantes University Hospital, Nantes, France.

Received: 4 February 2020 Accepted: 3 July 2020

Published online: 14 July 2020

\section{References}

1. de Kraker MEA, Wolkewitz M, Davey PG, Koller W, Berger J, Nagler J, et al. Burden of antimicrobial resistance in European hospitals: excess mortality and length of hospital stay associated with bloodstream infections due to
Escherichia coli resistant to third-generation cephalosporins. J Antimicrob Chemother. 2011;66(2):398-407.

2. Iredell J, Brown J, Tagg K. Antibiotic resistance in Enterobacteriaceae: mechanisms and clinical implications. BMJ. 2016;8:h6420.

3. Surveillance of antimicrobial resistance in Europe 2017 [Internet]. European Centre for Disease Prevention and Control. 2018 [cited 2019 Jan 9]. Available from: http://ecdc.europa.eu/en/publications-data/surveillanceantimicrobial-resistance-europe-2017.

4. Jean S-S, Hsueh P-R. SMART Asia-Pacific group. Distribution of ESBLs, AmpC $\beta$-lactamases and carbapenemases among Enterobacteriaceae isolates causing intra-abdominal and urinary tract infections in the Asia-Pacific region during 2008-14: results from the study for monitoring antimicrobial resistance trends (SMART). J Antimicrob Chemother. 2017:72(1):166-71.

5. Wilson APR. Sparing carbapenem usage. J Antimicrob Chemother. 2017; 72(9):2410-7.

6. Carlet J. The gut is the epicentre of antibiotic resistance. Antimicrob Resist Infect Control. 2012;1(1):39.

7. Shoemaker NB, Vlamakis H, Hayes K, Salyers AA. Evidence for extensive resistance gene transfer among Bacteroides spp. and among Bacteroides and other genera in the human colon. Appl Environ Microbiol. 2001;67(2):561-8.

8. Ubeda C, Taur Y, Jenq RR, Equinda MJ, Son T, Samstein M, et al. Vancomycin-resistant Enterococcus domination of intestinal microbiota is enabled by antibiotic treatment in mice and precedes bloodstream invasion in humans. J Clin Invest. 2010;120(12):4332-41.

9. Le Bastard Q, Al-Ghalith GA, Grégoire M, Chapelet G, Javaudin F, Dailly E, et al. Systematic review: human gut dysbiosis induced by non-antibiotic prescription medications. Aliment Pharmacol Ther. 2018;47(3):332-45.

10. Ubeda C, Bucci V, Caballero S, Djukovic A, Toussaint NC, Equinda M, et al. Intestinal microbiota containing Barnesiella species cures vancomycin-resistant Enterococcus faecium colonization. Infect Immun. 2013;81(3):965-73.

11. Caballero S, Kim S, Carter RA, Leiner IM, Sušac B, Miller L, et al. Cooperating Commensals Restore Colonization Resistance to Vancomycin-Resistant Enterococcus faecium. Cell Host Microbe. 2017;21(5):592-602 e4.

12. Gosalbes MJ, Vázquez-Castellanos JF, Angebault C, Woerther P-L, Ruppé E, Ferrús $M L$, et al. Carriage of Enterobacteria producing extended-Spectrum $\beta$-lactamases and composition of the gut microbiota in an Amerindian community. Antimicrob Agents Chemother. 2016;60(1):507-14.

13. Piewngam $P$, Quiñones $M$, Thirakittiwatthana $W$, Yungyuen $T$, Otto $M$, Kiratisin P. Composition of the intestinal microbiota in extended-spectrum $\beta$-lactamase-producing Enterobacteriaceae carriers and non-carriers in Thailand. Int J Antimicrob Agents. 2019;53(4):435-41.

14. Sorbara MT, Dubin K, Littmann ER, Moody TU, Fontana E, Seok R, et al. Inhibiting antibiotic-resistant Enterobacteriaceae by microbiota-mediated intracellular acidification. J Exp Med. 2019;216(1):84-98.

15. Birgand G, Hayatgheib N, Bémer P, Guilloteau V, Legeay C, Perron S, et al. Multi-drug-resistant Enterobacteriacae carriage in highly exposed nursing homes: prevalence in western France. J Hosp Infect. 2017:97(3):258-9.

16. Hillmann B, Al-Ghalith GA, Shields-Cutler RR, Zhu Q, Gohl DM, Beckman KB, et al. Evaluating the Information Content of Shallow Shotgun Metagenomics. mSystems. 2018;3(6) Available from: https://www.ncbi.nlm. nih.gov/pmc/articles/PMC6234283/. [cited 2019 Aug 1].

17. O'Leary NA, Wright MW, Brister JR, Ciufo S, Haddad D, McVeigh R, et al. Reference sequence (RefSeq) database at NCBI: current status, taxonomic expansion, and functional annotation. Nucleic Acids Res. 2016;44(D1):D733-45.

18. Vangay $\mathrm{P}$, Johnson AJ, Ward TL, Al-Ghalith GA, Shields-Cutler RR, Hillmann BM, et al. US Immigration Westernizes the Human Gut Microbiome. Cell. 2018;175(4):962-72 e10.

19. Gabriel Al-Ghalith, danknights. knights-lab/BURST: BURST v0.99.5a [Internet]. Zenodo; 2017 [cited 2019 Feb 8]. Available from: https://zenodo.org/ record/1037553.

20. Abubucker S, Segata N, Goll J, Schubert AM, Izard J, Cantarel BL, et al. Metabolic reconstruction for metagenomic data and its application to the human microbiome. PLoS Comput Biol. 2012;8(6):e1002358.

21. Caporaso JG, Kuczynski J, Stombaugh J, Bittinger K, Bushman FD, Costello EK, et al. QIIME allows analysis of high-throughput community sequencing data. Nat Methods. 2010;7(5):335-6.

22. Buchfink $B$, Xie C, Huson DH. Fast and sensitive protein alignment using DIAMOND. Nat Methods. 2015;12(1):59-60.

23. Lombard V, Golaconda Ramulu H, Drula E, Coutinho PM, Henrissat B. The carbohydrate-active enzymes database (CAZy) in 2013. Nucleic Acids Res. 2014;42(Database issue):D490-5. 
24. Love Ml, Huber W, Anders S. Moderated estimation of fold change and dispersion for RNA-seq data with DESeq2. Genome Biol. 2014;15(12):550.

25. Sauerbrei W, Schumacher M. A bootstrap resampling procedure for model building: application to the cox regression model. Stat Med. 1992;11(16): 2093-109.

26. Hillis DM, Bull JJ. An empirical test of bootstrapping as a method for assessing confidence in phylogenetic analysis. Syst Biol. 1993;42(2):182-92.

27. Morgan XC, Tickle TL, Sokol H, Gevers D, Devaney KL, Ward DV, et al. Dysfunction of the intestinal microbiome in inflammatory bowel disease and treatment. Genome Biol. 2012;13(9):R79.

28. Taur Y, Xavier JB, Lipuma L, Ubeda C, Goldberg J, Gobourne A, et al. Intestinal domination and the risk of bacteremia in patients undergoing allogeneic hematopoietic stem cell transplantation. Clin Infect Dis Off Publ Infect Dis Soc Am. 2012;55(7):905-14.

29. Vos P, Garrity G, Jones D, Krieg NR, Ludwig W, Rainey FA, et al., editors. Bergey's Manual of Systematic Bacteriology: Volume 3: The Firmicutes [Internet]. 2nd ed. New York: Springer-Verlag; 2009 [cited 2019 Mar 5]. (Bergey's Manual of Systematic Bacteriology). Available from: https://www. springer.com/us/book/9780387950419.

30. Nagai F, Watanabe Y, Morotomi M. Slackia piriformis sp. nov. and Collinsella tanakaei sp. nov., new members of the family Coriobacteriaceae, isolated from human faeces. Int J Syst Evol Microbiol. 2010;60(Pt 11):2639-46.

31. Belenguer A, Duncan SH, Calder AG, Holtrop G, Louis P, Lobley GE, et al. Two routes of metabolic cross-feeding between Bifidobacterium adolescentis and butyrate-producing anaerobes from the human gut. Appl Env Microbiol. 2006;72(5):3593-9.

32. Maathuis A, Hoffman A, Evans A, Sanders L, Venema K. The effect of the undigested fraction of maize products on the activity and composition of the microbiota determined in a dynamic in vitro model of the human proximal large intestine. J Am Coll Nutr. 2009;28(6):657-66.

33. Leo S, Lazarevic V, Gaïa N, Estellat C, Girard M, Matheron S, et al. The intestinal microbiota predisposes to traveler's diarrhea and to the carriage of multidrug-resistant Enterobacteriaceae after traveling to tropical regions. Gut Microbes. 2019;0(0):1-11.

34. Feehily C, Karatzas KAG. Role of glutamate metabolism in bacterial responses towards acid and other stresses. J Appl Microbiol. 2013;114(1):11-24.

35. Zhu L, Peng Q, Song F, Jiang Y, Sun C, Zhang J, et al. Structure and regulation of the gab gene cluster, involved in the gamma-aminobutyric acid shunt, are controlled by a sigma54 factor in bacillus thuringiensis. J Bacteriol. 2010;192(1):346-55.

36. Spiga L, Winter MG, Furtado de Carvalho T, Zhu W, Hughes ER, Gillis CC, et al. An Oxidative Central Metabolism Enables Salmonella to Utilize Microbiota-Derived Succinate. Cell Host Microbe. 2017;22(3):291-301 e6.

37. Ferreyra JA, Wu K, Hryckowian AJ, Bouley DM, Weimer BC, Sonnenburg JL. Gut microbiota-produced succinate Promotes $C$. difficile infection after antibiotic treatment or motility disturbance. Cell Host Microbe. 2014;16(6):770-7.

38. Alteri CJ, Smith SN, Mobley HLT. Fitness of Escherichia coli during urinary tract infection requires gluconeogenesis and the TCA cycle. PLoS Pathog. 2009;5(5):e1000448.

39. Bertin Y, Deval C, de la Foye A, Masson L, Gannon V, Harel J, et al. The Gluconeogenesis Pathway Is Involved in Maintenance of Enterohaemorrhagic Escherichia coli O157:H7 in Bovine Intestinal Content. PLoS ONE. 2014;9(6) Available from: https://www.ncbi.nlm.nih.gov/pmc/ articles/PMC4041753/. [cited 2019 Mar 28].

40. Ng KM, Ferreyra JA, Higginbottom SK, Lynch JB, Kashyap PC, Gopinath S, et al. Microbiota-liberated host sugars facilitate post-antibiotic expansion of enteric pathogens. Nature. 2013;502(7469):96-9.

41. Schwab C, Berry D, Rauch I, Rennisch I, Ramesmayer J, Hainzl E, et al. Longitudinal study of murine microbiota activity and interactions with the host during acute inflammation and recovery. ISME J. 2014;8(5):1101-14.

42. Barton LL, Ritz NL, Fauque GD, Lin HC. Sulfur cycling and the intestinal microbiome. Dig Dis Sci. 2017;62(9):2241-57.

\section{Publisher's Note}

Springer Nature remains neutral with regard to jurisdictional claims in published maps and institutional affiliations.

Ready to submit your research? Choose BMC and benefit from:

- fast, convenient online submission

- thorough peer review by experienced researchers in your field

- rapid publication on acceptance

- support for research data, including large and complex data types

- gold Open Access which fosters wider collaboration and increased citations

- maximum visibility for your research: over $100 \mathrm{M}$ website views per year

At BMC, research is always in progress.

Learn more biomedcentral.com/submissions 